\title{
EQUIVALENT IMPERFECTIONS IN ARCHED STRUCTURES
}

\author{
Marian DALLEMULE ${ }^{1 *}$
}

\section{Abstract}

There are currently three design methods to verify the in-plane buckling of an arched structure: substitute member method, the method of equivalent imperfection with recommendations for arched bridges, and the equivalent unique global and local initial imperfection method (EUGLI), which uses the critical elastic buckling mode as an imperfection. The latter method is included in the EN 1993-1-1 cl. 5.3.2 (11) since 2002; however, to this day it is neither utilized in the design practice nor is it incorporated in ordinary structural analysis software. The main purpose of this article is to show the application of the proposed methods in a step-by-step manner to the numerical example considered and to compare these design methods for various arched structures. Verification of the in-plane buckling of an arch is explained in detail.

\section{Address}

1 Ingsteel spol. s r. O., Tomášikova 17, P. O. Box 82, 820 09, Bratislava

Department of Steel and Timber Structures, Faculty of Civil Engineering, Slovak University of Technology in Bratislava, Radlinského 11, 81368 Bratislava, Slovakia

* Corresponding author: dallemule@ingsteel.sk, marian.dallemule@stuba.sk

\section{Key words}

- Arches,

- Imperfections,

- Buckling mode,

- EUGLI method.

\section{INTRODUCTION}

Arched structures are often used as supporting structures for bridges or other long-span structures. A well-designed arch should be purely under compression, but in the real world, it is also bent due to the nature of a live load or various imperfections. From a design point of view, arches are complicated and interesting and are usually a part of important civil structures. Verification of an in-plane buckling of an arched structure (Fig. 1) is provided, using three different design methods according to the Eurocodes:

1) The substitute member method (SM) according to (STN EN 1993-1-1)

2) Equivalent imperfections for arched bridges (TAB. D.8) according to STN EN 1993-2 Annex D, D.3, Table D.8

3) The Equivalent Unique Global and Local Initial imperfection method (EUGLI) according to (STN EN 1993-1-1) cl. 5.3.2 (11)

A comparative parametric study was carried out for hingeless (fixed), 3-hinge and 2-hinge arches.

\section{THE NUMERICAL EXAMPLE CONSIDERED}

Static and buckling analyses were carried out using the $1^{\text {st }}$ order theory for the $S M$ method and the $2^{\text {nd }}$ order theory for TAB. D. 8 and the EUGLI method. The $2^{\text {nd }}$ order analysis used in this article is not in fact a large deflection analysis - the so-called Newton-Raphson method (in terms of numerical analysis) - but a simplified approach, which only takes into account only the contribution of axial forces on the displacements. The difference in using this simplified approach and a large deflection analysis is negligible for ordinary frame structures. The graphic results for both theories are similar, almost identical in shape, and can be seen in Fig. 2. For the numerical results, see Table 1 .

$$
\begin{array}{ll}
\text { Arch: } & \mathrm{L}=40 \mathrm{~m} ; \mathrm{f}=8 \mathrm{~m} ; \mathrm{L}_{\text {real }}=43.93 \mathrm{~m} ; \text { shape }=\text { parabola } \\
\text { Load: } & \mathrm{g}_{\mathrm{Ed}, 1}=48 \mathrm{kN} / \mathrm{m} ; \mathrm{g}_{\mathrm{Ed}, 2}=36 \mathrm{kN} / \mathrm{m} \\
\text { Material: } & \mathrm{f}_{\mathrm{y}}=275 \mathrm{MPa} ; \mathrm{g}_{\mathrm{M} 1}=1.1 ; \mathrm{E}=210 \mathrm{GPa} \\
\text { Cross-section: } & \mathrm{A}=0.03 \mathrm{~m}^{2} ; \mathrm{I}_{\mathrm{y}}=1.2186 \cdot 10^{-3} \mathrm{~m}^{4} ; \\
& \mathrm{W}_{\text {el, }}=5.0775 \cdot 10^{-3} \mathrm{~m}^{3} ; \mathrm{a}_{\mathrm{y}}=0.49 \\
& \mathrm{CS}=\text { class } 3 ; \\
& \mathrm{CS} \text { is not susceptible to lateral-torsional buckling }
\end{array}
$$




\section{$g_{E d, 1} \sqrt{\downarrow+\downarrow \downarrow \downarrow \downarrow \downarrow \downarrow \downarrow \downarrow+1+1+1+\downarrow} g_{E d, 2}$}

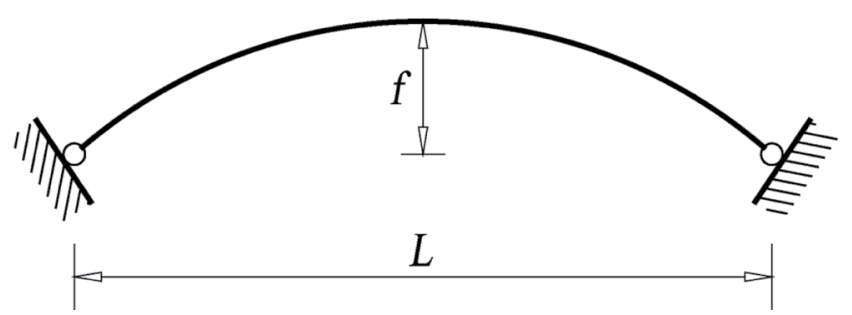

Fig. 1 Static scheme of the example given.

The arch was divided into 80 elements with 81 nodes for the FEM analysis. The elements were numbered $1 \sim 80$ from left to right. The nodes were numbered as follows: the leftmost node is No. 1; the topmost node (at the crown) is No. 2; and the rightmost node is No. 3. The nodes on the left side are numbered from left to right as 4 42 and on the right side as $43 \sim 81$. The uniform loading was replaced with nodal loads in a vertical direction (plane):

$$
\begin{aligned}
& Q_{E d, 1}=12 \mathrm{kN} ; Q_{E d, 4 \sim 42}=24 \mathrm{kN} ; Q_{E d, 2}=21 \mathrm{kN} ; \\
& Q_{E d, 43 \sim 81}=18 \mathrm{kN} ; Q_{E d, 3}=9 \mathrm{kN} .
\end{aligned}
$$

Note: Based on the buckling analysis the $1^{\text {st }}$ buckling mode $h_{c r}(x)$ is obtained. The buckling mode is usually normalized through one of its displacement components, either $u$ (the horizontal component), $v$ (the vertical component) or $\eta$ (the nodal rotation), whichever is greatest. For the EUGLI method purposes, the buckling mode is normalized based on the maximum absolute vector size $\left|\eta_{c r}\right|_{\max }$.

This is achieved in the example at node No. 22: $\left|\eta_{c r, 22}\right|=1.000$, where $\eta_{c r, 22, u}=0.554$ and $\eta_{c r, 22, v}=-0.833$.

\section{VERIFICATION}

\subsection{Substitute Member Method}

The substitute member method is the most used method for verifying compressed members. It was derived for members with a uniform axial force and cross-section throughout their lengths. This condition is not satisfied in the example, but the values of the axial

\begin{tabular}{|c|c|c|c|c|}
\hline \multirow[b]{2}{*}{ Quantity } & \multicolumn{2}{|c|}{$1^{\text {st }}$ order analysis } & \multicolumn{2}{|c|}{$2^{\text {nd }}$ order analysis } \\
\hline & value & node / elem* & value & node / elem* \\
\hline$R_{H, L}$ & 1048.69 & 1 & 1050.20 & 1 \\
\hline$R_{H, R}$ & \begin{tabular}{|l|}
1048.69 \\
\end{tabular} & 3 & 1050.20 & 3 \\
\hline$R_{V, L}$ & 900.00 & 1 & 898.51 & 1 \\
\hline$R_{V R}$ & 780.00 & 3 & 781.49 & 3 \\
\hline$N_{E d \text { max }} \mathrm{kN}$ & -1373.35 & $1^{*}$ & -1373.62 & $1^{*}$ \\
\hline$V_{E d, \max } \mathrm{kN}$ & -58.51 & $41^{*}$ & -71.13 & $41^{*}$ \\
\hline$M_{E d, \max } \quad$ kN.m & 307.88 & 23 & 397.25 & 23 \\
\hline$d e f_{\max } \mathrm{mm}$ & 69.367 & 23 & 88.064 & 23 \\
\hline$\eta_{c r}$ & 4.3936 & - & 4.3886 & - \\
\hline
\end{tabular}

Tab. 1 Short list of the results.

* According to the FEM analysis, the axial and shear forces are uniform along the element, the number for which represents the element's ordinal number. The other results are unique for each node, such as the reactions, bending moments, displacements, and buckling modes (displacements), in which case the number represent the node's ordinal number. These numbers are subsequently written as subscripts. To simplify the verification further, the values of the axial force in the adjacent elements could be averaged as if they were represented at a node. No simplification was performed in these calculations; thus for the verification, it has to be distinguished for each element as to which node is verified. This is irrelevant for the $S M$ method.

force change very slightly over the member's length; therefore, this method can be used for a comparison with a relatively good degree of accuracy.

The effect of any imperfection is hidden in the reduction factor $\chi$ through the imperfection factor, which in this case is $\alpha_{y}=0.49$ (buckling curve ' $\mathrm{c}$ ' for the given cross-section). All the internal forces are calculated using the $1^{\text {st }}$ order analysis. The $N_{E d \text { max }}$ and $M_{E d \text {,max }}$ are taken into account with the proper interaction factor $k_{y,}$, because they are not in the same position. Using $\alpha_{c r}$ and $N_{E d, \max }$, the quantities $\lambda_{\text {rel }}, \chi$ and $N_{b, R d}$ (the design buckling resistance of a member) could be determined.

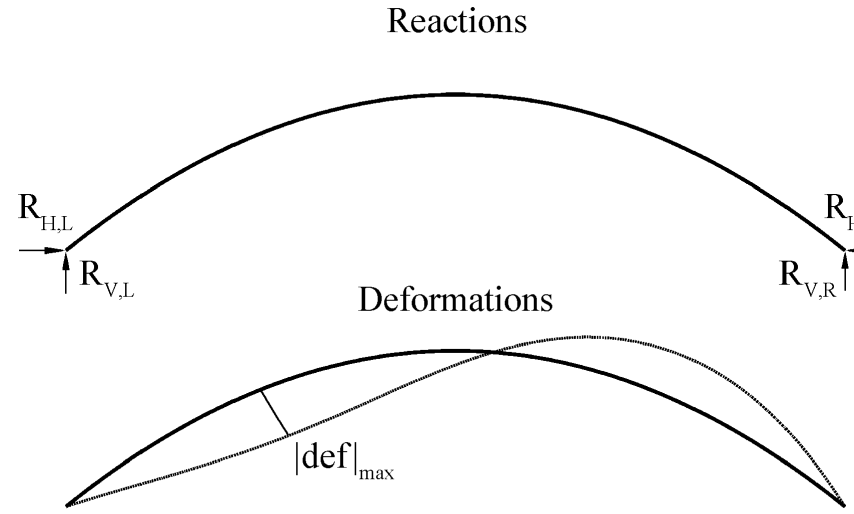

Buckling mode

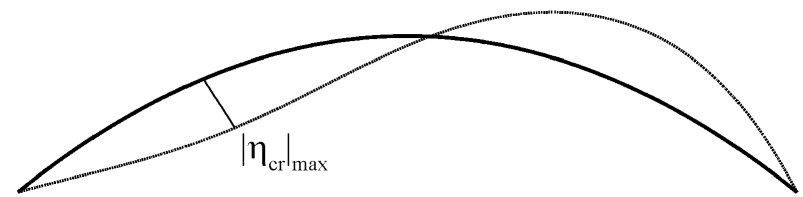

Axial forces

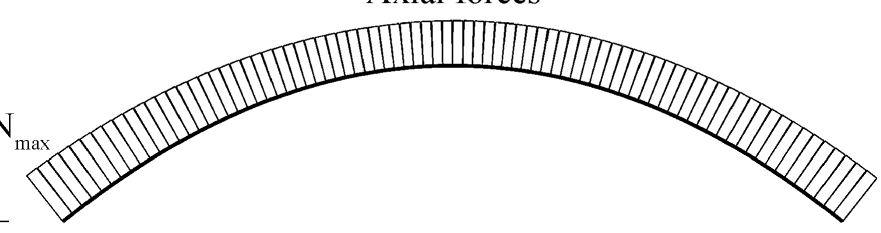

Shear forces

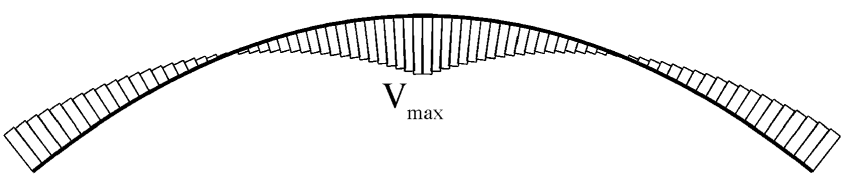

Bending moments

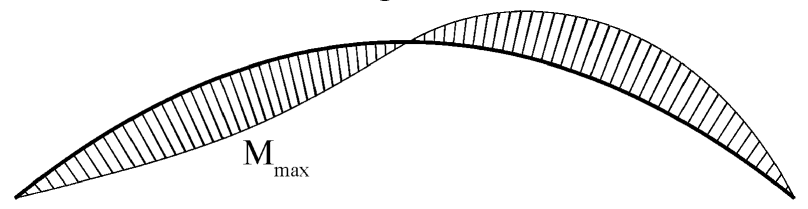

Fig. 2 Results of the static and buckling analyses $\left(N_{\max }=N_{E d, \max } ; V_{\max }=V_{E d, \max } ; M_{\max }=M_{E d, \max }\right)$. 
$\lambda_{\text {rel }}=\sqrt{\frac{A \cdot f_{y}}{\alpha_{c r} \cdot N_{E d, \max }}}=\sqrt{\frac{0.03 \cdot 275000}{4.3936 \cdot 1373.35}}=1.169$

$\phi=0.5 \cdot\left(1+\alpha_{y} \cdot\left(\lambda_{\text {rel }}-0.2\right)+\lambda_{\text {rel }}{ }^{2}\right)=0.5 \cdot\left(1+0.49 \cdot(1.169-0.2)+1.169^{2}\right)=1.421$

$\chi=1 /\left(\phi+\sqrt{\phi^{2}-\lambda_{\text {rel }}^{2}}\right)=1 /\left(1.421+\sqrt{1.421^{2}-1.169^{2}}\right)=0.449$

$N_{b, R d}=\chi \cdot A \cdot \frac{f_{y}}{\gamma_{M 1}}=0.449 \cdot 0.03 \cdot \frac{275000}{1.1}=3365.12 \mathrm{kN}$

$k_{y y}=C_{m y} \cdot\left(1+0.6 \cdot \lambda_{r e l} \cdot \frac{N_{E d, \max }}{N_{b, R d}}\right)=0.9 \cdot\left(1+0.6 \cdot 1.169 \cdot \frac{1373.35}{3365.12}\right)=1.1577$

$U=\frac{N_{E d, \text { max }}}{N_{b, R d}}+k_{y y} \cdot \frac{M_{E d, \text { max }}}{M_{R d}}=\frac{1373.35}{3365.12}+1.1577 \cdot \frac{307.88}{1269.38}=0.6889<1$

\subsection{Equivalent Imperfections For Arched Bridges}

An equivalent imperfection for an arched structure could be obtained according to STN EN 1993-2, annex D, chapter D.3 Arched bridges, Table 8. Shape and amplitudes of imperfections for in-plane buckling of arches. When dealing with imperfections, internal forces have to be calculated using the $2^{\text {nd }}$ order analysis. The equivalent imperfection has the form of two asymmetric half-waves of the sine function - see Table 2. The amplitude for this type of imperfection depends on the buckling curve assigned for the given cross-section. In our case (buckling curve "c"), $e_{0}= \pm L / 400= \pm 40 / 400= \pm 0.1 \mathrm{~m}$.

Tab. 2 Table D.8, taken from STN EN 1993-2 Annex D.

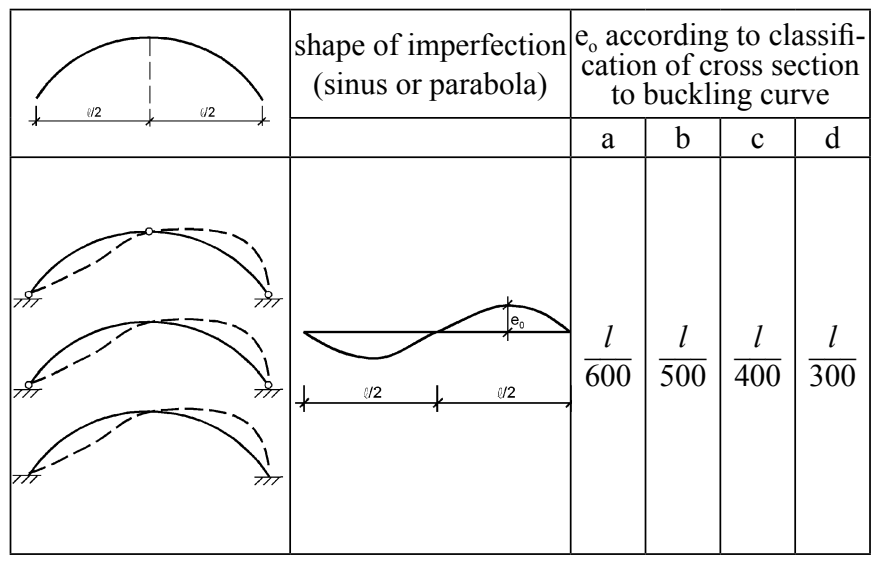

The imperfection could be (for the sake of the simplification) replaced by a uniform asymmetric load along the arch - see (1).

$$
q_{e q}= \pm \frac{8 \cdot R_{H} \cdot e_{0}}{(0.5 \cdot L)^{2}}= \pm \frac{8 \cdot 1050.20 \cdot 0.1}{(0.5 \cdot 40)^{2}}=2.1 \mathrm{kN} / \mathrm{m}
$$

Taking into account the imperfection, the equivalent design uniform load is:

$$
\begin{aligned}
& g_{E d, 1, e q}=g_{E d, 1}+q_{e q}=48+2.1=50.1 \mathrm{kN} / \mathrm{m} \\
& E d, 2, e q \\
& =g_{E d, 2}-q_{e q}=36-2.1=33.9 \mathrm{kN} / \mathrm{m}
\end{aligned}
$$

Note: The difference in internal forces between using the equivalent load and the original load acting on an imperfect structure is negligible.

The maximum stress has been achieved at element 19, node No. 22, where:

$$
U=\frac{N_{E d, 19}}{N_{R d}}+\frac{M_{E d, 22}}{M_{R d}}=\frac{1144.97}{7500}+\frac{533.37}{1269.375}=0.5728<1
$$

\subsection{The Eugli Imperfection Method}

The Equivalent Unique Global and Local Initial Imperfection (sometimes referred as the "EUGLI imperfection") is a relatively new design method that has not been well utilized in design practice. It can be found in (STN EN 1993-1-1) clause 5.3.2 (11). This method will be described below.

The main idea is that the shape of the imperfection has the form of the critical elastic buckling mode. The $S M$ method is also based on the same idea, where the imperfection for a simply supported member with a uniform cross-section and axial force all over the length of the member has the shape of a half-wave of the sine function. Chladný (2013) generalized this procedure for frame structures. This method is also designated for the verification of members with a non-uniform cross-section and/or non-uniform axial compressive force Kováč (2012). In recent times, it was successfully used in the design of two similar arched bridges, i.e., the Apollo bridge (Slovakia 2005) and the Pentele bridge (Hungary 2007).

The advantage is that the equivalent imperfection includes both global and local aspects for the verification of stability. This shape is considered to be the most effective shape for the loss of stability in general. Other methods according to (STN EN 1993-1-1) 5.3.2 separate local and global imperfections in terms of a local bow imperfection and the initial sway of columns (for frame structures). Almost all of the structural analysis software today provides stability calculations, which results in $\alpha_{c r}$ and the shape of the critical elastic buckling mode $\eta_{c r}(x)$. Both of these results are necessary not only for this method to be used but also for the $2^{\text {nd }}$ derivation of $\eta_{c r}(x)$, which, however, most of the software does not provide, but that is just a matter of a numerical evaluation. The problem with this verification approach is that there are no experimentally established and statistically evaluated amplitudes for these types of imperfections. Considering the complexity of frame structures, it is probably not possible. Because no amplitudes have been established, it is possible to use the ones determined by the Basic requirement, i.e., The buckling resistance of a frame structure with axially loaded members shall be equal to the flexural buckling resistance of the substitution member (Chladný, 2013). This premise is the main contribution of Chladny's paper.

The imperfection shape $\eta_{\text {initm }}(x)$ shall be affine to the $1^{\text {st }}$ buckling mode $\eta_{c r}(x)$. Its maximum amplitude $\eta_{\text {initm,max }}$ ( $m$ is the critical cross-section) is based on $e_{0, d}$ (the design value of the initial bow imperfection) and $M_{\eta, c r m}$ (the bending moments due to the shape of $\left.\eta_{\text {init, }}(x)\right)$; see an illustrative example in Fig. 3.

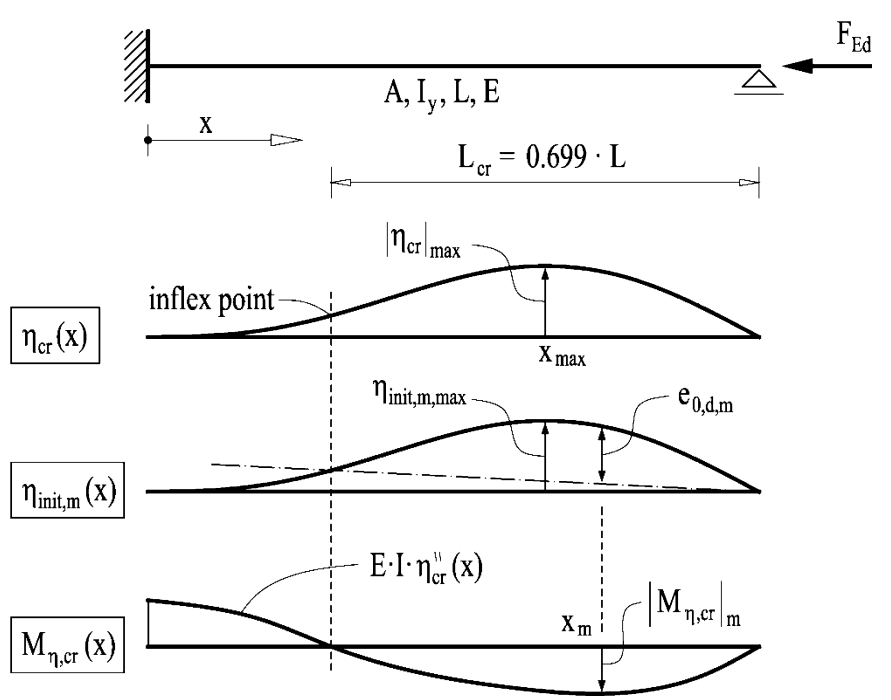

Fig. 3 An illustrative example. Note the 'hidden'location of $e_{0, d, m .}$ 
The results of the Basic requirement are that if the utilization factor of the member is less than 1.0 according to the $S M$ method, then, according to the EUGLI method, the utilization factor for the same member is even lower. In the event the utilization factor of the member is equal to 1.0 according to the $S M$ method, then it is also equal to 1.0 according to the EUGLI method. This is caused by the behaviour of the $2^{\text {nd }}$ order analysis. The equivalent initial imperfection $\eta_{i n i t, m}(x)$ is determined by multiplying the shape $\eta_{c r}(x)$ with the maximum amplitude $\eta_{\text {init,m,max }}$. It covers both the global and local imperfections for the entire structure. From the imperfect structure analysis, the addition of bending moments $M_{\eta, c r}(x)$ (as an influence of the axial force acting on an imperfect structure) can be clearly separated from the bending moments due to the external load. Note that $e_{0, d, m}$ is at the point where the curvature of $\eta_{\text {init, } m}(x)$ is at its maximum, and it can be graphically interpreted, as can be seen in Fig. 3. The cross-section verification, instead of the member verification, can be carried out. All the internal forces should be calculated using the $2^{\text {nd }}$ order analysis. This method is iterative in general, because the critical cross-section $x_{m}$ has to be found. It is the point where the interaction of $N_{E d, m}$ and $M_{E d, m}+M_{\eta, \text { init, }, m}$ has the overall maximum effect. There are four combinations in the relation between the uniform or non-uniform axial force and the uniform or non-uniform cross-section. Only in the case where both the crosssection and axial force are uniform is the calculation not iterative; the position of the critical cross-section $x_{m}$ could be found directly.

The reader can find more information and examples developed in Baláž (2008), Baláž (2009), Baláž and Koleková (2010) and Baláž et al. (2010). The basic formulae with the description for the EUGLI method (taken from the references) are listed below:

Characteristic value of the equivalent initial bow imperfection:

$$
e_{0, k, m}=\alpha \cdot\left(\bar{\lambda}_{m}-0.2\right) \cdot \frac{M_{R k, m}}{N_{R k, m}}
$$

Design value of the equivalent initial bow imperfection:

$$
e_{0, d, m}=e_{0, k, m} \cdot \delta_{e}
$$

where:

$$
\delta_{e}=\frac{1-\chi \cdot \bar{\lambda}_{m}^{2} / \gamma_{M 1}}{1-\chi \cdot \bar{\lambda}_{m}^{2}}
$$

The bending moment in the critical cross-section $m$ due to the $\eta_{c r}(x)$ is:

$$
\left|M_{\eta, c r}\right|_{m}=E . I_{m} \cdot\left|\eta_{c r}{ }^{\prime \prime}(x)\right|_{m}
$$

The design value of the maximum amplitude for the EUGLI imperfection $\eta_{\text {init, }}(x)$ :

Tab. 3 Results.

$$
\eta_{\text {init,m,max}}=\frac{\alpha_{c r} \cdot e_{0, d, m} \cdot N_{E d, m}}{\left|M_{\eta, c r}\right|_{m}} \cdot\left|\eta_{c r}\right|_{\max }
$$

The shape of the $\eta_{\text {init, }}(x)$ imperfection:

$$
\eta_{\text {init,m }}(x)=\eta_{\text {init,m,max }} \cdot \frac{\eta_{c r}(x)}{\left|\eta_{c r}\right|_{\max }}
$$

Bending moments on the imperfect structure due to the action of axial force $N_{E d}$ :

$$
M_{\eta, \text { init,m }}(x)=-E . I(x) \cdot \frac{\eta_{\text {init, } m, \max }}{\alpha_{c r}-1} \cdot \frac{\eta_{c r}{ }^{\prime \prime}(x)}{\left|\eta_{c r}\right|_{\max }}
$$

If the critical point $m$ could be located directly, (8) could be simplified into (9):

where:

$$
M_{\eta, i n i t, m, m}=k \cdot e_{0, d, m} \cdot N_{E d, m}
$$

$\left|\eta_{c r}\right|_{\max }$ is the maximum amplitude of the critical buckling mode, which usually equals 1.0 if the buckling mode is normalized; $\bar{\lambda}_{m}$ is the relative slenderness for the critical cross-section $m ; \alpha$ is the imperfection factor for the relevant buckling curve; and $\alpha_{c r}$ is the minimum force amplifier for the axial force configuration $N_{E d}^{c r}$ in the members to reach the critical elastic force.

The verification according to the $E U G L I$ method could be divided into five steps:

1) After carrying out the static and buckling analyses, the curvature of the buckling mode $\eta_{c r}$ " $(x)$ should be calculated. These calculations can be done 'by hand' or by using software, e.g. Rubin et al. (2004) (the author does not know of any other software that can provide these calculations). 'By hand' means to use one of the methods of numerical differentiation, e.g. (20). To obtain the curvature of $\eta_{c r}(x)$ either the $2^{\text {nd }}$ derivation of the displacements or the $1^{\text {st }}$ derivation of the rotations has to be performed. Using the displacements in the case of arches is questionable due to the nature of the node displacement. A node displacement of a frame structure (in general) always has two components (vertical and horizontal) versus a single member - where this question would never arise. The rotation of the nodes, which is already the $1^{\text {st }}$ derivation of $\eta_{c r}(x)$, is known from the buckling analysis too. To obtain the required $\eta_{c r}$ "' $(x)$, a differentiation of these rotations is needed. This method is considered to be less accurate in general as opposed to the $2^{\text {nd }}$ differentiation of the displacements, but it seems to be less questionable too. It is advisable to use the second

\begin{tabular}{|c|c|c|c|c|c|c|c|c|c|c|}
\hline elem & node & $\mathrm{N}_{\mathrm{Ed}}$ & $\mathrm{M}_{\mathrm{Ed}}$ & $\mathrm{M}_{\eta, \mathrm{cr}}$ & $\mathrm{U}_{\mathrm{N}+\mathrm{M}}$ & $\lambda_{\text {rel }}$ & $\chi$ & $\delta_{\mathrm{e}}$ & $e_{o d}$ & $\eta_{\text {init,m,max }}$ \\
\hline \multirow{2}{*}{17} & 19 & \multirow{2}{*}{1164.21} & 384.08 & 4.805 & 0.4578 & 1.271 & 0.401 & 1.167 & 0.104 & 32.528 \\
\hline & 20 & & 390.44 & 4.836 & 0.4628 & 1.271 & 0.401 & 1.167 & 0.104 & 32.320 \\
\hline \multirow{2}{*}{18} & 20 & \multirow{2}{*}{1154.07} & 390.44 & 4.836 & 0.4615 & 1.276 & 0.399 & 1.169 & 0.104 & 32.241 \\
\hline & 21 & & 394.75 & 4.837 & 0.4649 & 1.276 & 0.399 & 1.169 & 0.104 & 32.234 \\
\hline \multirow{2}{*}{19} & 21 & \multirow{2}{*}{1144.33} & 394.75 & 4.837 & 0.4636 & 1.282 & 0.397 & 1.170 & 0.105 & 32.157 \\
\hline & 22 & & 397.01 & 4.810 & 0.4653 & 1.282 & 0.397 & 1.170 & 0.105 & $\underline{32.343}$ \\
\hline \multirow{2}{*}{20} & 22 & \multirow{2}{*}{1135.02} & 397.01 & 4.810 & 0.4641 & 1.287 & 0.394 & 1.171 & 0.106 & 32.268 \\
\hline & 23 & & 397.25 & 4.754 & 0.4643 & 1.287 & 0.394 & 1.171 & 0.106 & 32.643 \\
\hline
\end{tabular}
approach, i.e., differentiating the rotations.

$$
y_{i}^{\prime} \approx \frac{y_{i+1}-y_{i-1}}{2 \cdot h}
$$

$y_{i}{ }^{\prime}$ is in fact the $\eta_{c r}{ }^{\prime \prime}(x) ; y$ is the node rotation; $h$ is the distance between two adjacent nodes.

Note: To preserve accuracy, all the distances (length of the elements) should be equal. 
2) The cross-section at each element has a different axial force for which it is necessary to calculate quantities $\lambda_{r e l}$ and $e_{0, d}$. Because the $M_{\eta c r}$ is different for each node, $\eta_{\text {init,m,max }}$ has to be calculated for both ending nodes of an element - see the results in Table 3.

For the sake of clarity, a representative calculation for element 19 , node 22 , is provided below:

$$
\begin{aligned}
& \begin{aligned}
M_{\eta, c r, 22}=E \cdot I \cdot\left|\eta_{c r}^{\|}\left(x_{22}\right)\right|=210 \cdot 10^{6} \cdot 1.2186 \cdot 10^{-3} \cdot 0.018794 \cdot 10^{-3}= \\
=4.810 \mathrm{kN} \cdot \mathrm{m}
\end{aligned} \\
& \begin{aligned}
k=\frac{1}{1-1 / \alpha_{c r}}=\frac{1}{1-1 / 4.3886}=1.2951 \\
U_{N 19+M 22}=\frac{N_{E d}}{N_{R d}}+\frac{M_{E d}}{M_{R d}}=\frac{N_{E d, 19}}{A \cdot f_{y} / \gamma_{M 1}}+\frac{M_{E d, 22}}{W_{e l, y} \cdot f_{y} / \gamma_{M 1}} \\
U_{N 19+M 22}=\frac{1144.33}{0.03 \cdot 275 \cdot 10^{3} / 1.1}+\frac{397.01}{5.0775 \cdot 10^{-3} \cdot 275 \cdot 10^{3} / 1.1}= \\
=0.1526+0.3127=0.4653
\end{aligned}
\end{aligned}
$$$$
\lambda_{r e l, 19}=\sqrt{N_{R k} / N_{c r, 19}}=\sqrt{\frac{A \cdot f_{y}}{\alpha_{c r} \cdot N_{E d, 19}}}=\sqrt{\frac{0.03 \cdot 275 \cdot 10^{3}}{4.3886 \cdot 1144.33}}=1.282
$$$$
\phi_{19}=0.5 \cdot\left(1+\alpha \cdot\left(\lambda_{\text {rel }, 19}-0.2\right)+\lambda_{\text {rel }, 19}^{2}\right)=
$$$$
=0.5 \cdot\left(1+0.49 \cdot(1.282-0.2)+1.282^{2}\right)=1.586
$$$$
\chi_{19}=1 /\left(\phi_{19}+\sqrt{{\phi_{19}{ }^{2}-\lambda_{\text {rel }, 19}{ }^{2}}^{2}}\right)=1 /\left(1.586+\sqrt{1.586^{2}-1.282^{2}}\right)=0.397
$$$$
e_{0, d, 19}=e_{0, k} \cdot \delta_{e, 19}=\alpha \cdot\left(\lambda_{r e l, 19}-0.2\right) \cdot \frac{M_{R k}}{N_{R k}} \cdot \frac{1-\chi_{19} \cdot \lambda_{r e l, 19}^{2} / \gamma_{M 1}}{1-\chi_{19} \cdot \lambda_{r e l, 19}^{2}}
$$$$
e_{0, d, 19}=0.49 \cdot(1.282-0.2) \cdot \frac{5.0775 \cdot 10^{-3} \cdot 275 \cdot 10^{3}}{0.03 \cdot 275 \cdot 10^{3}} \cdot \frac{1-0.397 \cdot 1.282^{2} / 1.1}{1-0.397 \cdot 1.282^{2}}=
$$$$
=0.105
$$$$
\eta_{\text {init,m,19,22 }}=\frac{k \cdot e_{0, d, 19} \cdot N_{E d, 19}}{M_{\eta, c r, 22}}=\frac{1.2951 \cdot 0.105 \cdot 1144.33}{4.810}=32.343
$$

3) If the results are prepared, the critical cross-section $m$ for the first iteration could be estimated. Because the shape of the axial forces is changing very slightly over the length and because both maximum bending moments, i.e., $M_{E d, \max }$ and $M_{\eta, c r m a x}$, are located

Tab. 4 Results for the $1^{\text {st }}$ iteration.

\begin{tabular}{|c|c|c|c|c|}
\cline { 2 - 5 } \multicolumn{2}{c|}{} & \multicolumn{3}{c|}{$1^{\text {st }}$ iteration: $\eta_{\text {init,m,max }}=32.343$} \\
\hline \multirow{2}{*}{ elem } & node & $\eta_{\text {init,m,max }}$ & $\mathrm{M}_{\eta, \text { init }}$ & $\mathrm{U}$ \\
\hline \multirow{2}{*}{17} & 19 & 32.528 & 155.42 & 0.5802 \\
\cline { 2 - 5 } & 20 & 32.320 & 156.42 & 0.5860 \\
\hline \multirow{2}{*}{18} & 20 & 32.241 & 156.42 & 0.5847 \\
\cline { 2 - 5 } & 21 & 32.234 & 156.45 & $\mathbf{0 . 5 8 8 1}$ \\
\hline \multirow{2}{*}{19} & 21 & 32.157 & 156.45 & 0.5868 \\
\cline { 2 - 5 } & 22 & $\mathbf{3 2 . 3 4 3}$ & 155.55 & 0.5879 \\
\hline \multirow{2}{*}{20} & 22 & 32.268 & 155.55 & 0.5866 \\
\cline { 2 - 5 } & 23 & 32.643 & 153.76 & 0.5854 \\
\hline
\end{tabular}

approximately at the first quarter of the span, the location of point $m$ is also expected at this location, i.e., somewhere between nodes 19 23. But it is generally best to follow the stresses or the utilization factors $U$ (as in this case) and to choose the point with the maximum stress due to the $N_{E d}+M_{E d}$. Therefore, the element 19, node 22, has been chosen for the $1^{\text {st }}$ iteration step, $U_{N 19+M 22}=0.4653$, which is the maximum value of $U_{N+M}$.

4) The $1^{\text {st }}$ iteration: Based on the value $\eta_{\text {init,m,19,22 }}, M_{\eta, \text { init, } m}$ is calculated for the surrounding nodes. A point with the highest utilization factor (or stress) has to be found; see Table 4 .

There is also another approach that could be used to find this ultimate point. Using formula (7) the exact form of the imperfection for the given $\eta_{\text {init } m}$ is obtained. Executing the $2^{\text {nd }}$ order static analysis on a specific imperfect structure will result in a verification based only on the stresses. The disadvantage is that the bending moments $M_{E d}$ and additional $M_{\eta i n i t}$ cannot be separated in the verification. This approach is not used in this article.

Element 18, node 21, has the highest $U$, for which the calculations are as follows:

$M_{\eta, \text { init }, 18,21}=\eta_{\text {init, }, 19,22} \cdot M_{\eta, c r, 21}=32.343 \cdot 4.837=156.45 \mathrm{kN} \cdot \mathrm{m}$

$U=\frac{N_{E d, 18}}{N_{R d}}+\frac{M_{E d, 21}+M_{\eta, \text { init } 18,21}}{M_{R d}}=\frac{1154.07}{7500}+\frac{394.75+156.45}{1269.375}=0.5881$

5) Next iteration: Because the cross-section with the highest $U$ (element 18 , node 21 ) is not the same as the one initially expected (element 19, node 22), step 4 has to be repeated until they are the same. Now, based on the new value $\eta_{\text {init } m_{1} 18,21}$, the $M_{\eta \text { init, }}$ is calculated for the surrounding nodes. For the $2^{\text {nd }}$ iteration results, see Table 5.

Element 18, node 21, has the highest $U$, for which the calculations are as follows:

$M_{\eta, \text { init }, 18,21}=\eta_{\text {init,m,18,21 }} \cdot M_{\eta, c r, 18}=32.234 \cdot 4.837=155.92 \mathrm{kN} . \mathrm{m}$

$U=\frac{N_{E d, 18}}{N_{R d}}+\frac{M_{E d, 21}+M_{\eta, \text { init } t, 18,21}}{M_{R d}}=\frac{1154.07}{7500}+\frac{394.75+155.92}{1269.375}=0.5877<1$

The node with the highest utilization factor is the same as expected at the end of the previous iteration step. This verification converged quickly, i.e., after just two iterations, because in this case, the axial force is almost uniform; it changes very slightly along the structure. The critical point $m$ is usually 'moved' from the point of the maximum $U_{M}$ to the point of the maximum $U_{N}$. The $M_{\eta^{\prime} \text {, init }}$ and the final verification can be interpreted graphically, as seen in Fig. 4.

Tab. 5 Results for the $2^{\text {nd }}$ iteration.

\begin{tabular}{|c|c|c|c|c|}
\cline { 3 - 5 } \multicolumn{2}{c|}{} & \multicolumn{3}{c|}{$2^{\text {nd }}$ iteration: $\eta_{\text {init,m,max }}=32.234$} \\
\hline \multirow{2}{*}{ elem } & node & $\eta_{\text {init,m,max }}$ & $\mathrm{M}_{\eta, \text { init }}$ & $\mathrm{U}$ \\
\hline \multirow{2}{*}{17} & 19 & 32.528 & 154.89 & 0.5798 \\
\cline { 2 - 5 } & 20 & 32.320 & 155.89 & 0.5856 \\
\hline \multirow{2}{*}{18} & 20 & 32.241 & 155.89 & 0.5843 \\
\cline { 2 - 5 } & 21 & $\mathbf{3 2 . 2 3 4}$ & 155.92 & $\mathbf{0 . 5 8 7 7}$ \\
\hline \multirow{2}{*}{19} & 21 & 32.157 & 155.92 & 0.5864 \\
\cline { 2 - 5 } & 22 & 32.343 & 155.03 & 0.5875 \\
\hline \multirow{2}{*}{20} & 22 & 32.268 & 155.03 & 0.5862 \\
\cline { 2 - 5 } & 23 & 32.643 & 153.25 & 0.5850 \\
\hline
\end{tabular}


Bending moments due to the EUGLI imperfection

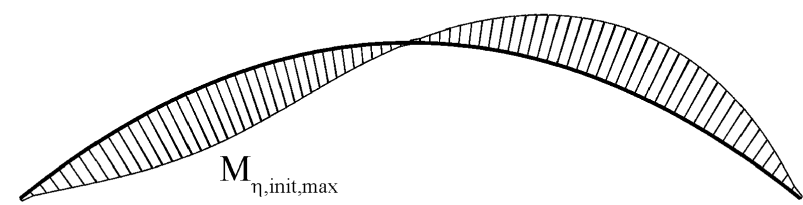

Fig. 4 The final verification.

\section{DISCUSSION AND CONCLUSION}

The comparison of these three design methods according to the Eurocodes has been carried out for the spectrum of $f / L=0.1 \sim 0.5$ for various arches: hingeless (Fig. 5), 2-hinge (Fig. 6) and 3-hinge (Fig. 7).

Comparing Figs. 5 7, the best 'match' in the verification using the three different design methods is for the hingeless arches. The differences are almost negligible. For the 2-hinge and 3-hinge arches, the substitute member method is about $10-20 \%$ on the 'safe' side compared to the equivalent imperfection methods. The impossibility of determining the ultimate cross-section position (point $\mathrm{m}$ ) in a structure is a drawback of the $S M$ method. For the 3-hinge arches, there is a really good 'match' in the verification between the Tab. $D .8$ method and the EUGLI method, which is caused by the fact that there are no bending moments due to the external load, because of the nature of the load relative to the static scheme and the shape of the arches. It has to be noted that none of these methods could be used for the verification of flat arches, i.e., a snap-through of arches. For considering such a phenomenon, there is a condition in [2], annex D, statement (D.5) and Tab. D.5. No snap-through analysis is needed for the given example, not even for arches with $f / L=0.1$.

Among these three design methods, the EUGLI method seems to be the most complicated, but it describes the buckling behavior in the best way. It also has the most efficient shape for the loss of stability, compared to the other equivalent imperfection methods. The iterative verification process would be exactly the same for members with a non-uniform cross-section as described in section 2.3. The bending and axial stiffness in this case should be different but uniform for each element. The procedure is best suited for use with structural software, where the buckling analysis is provided. Using this method, the addition of the bending moments due to the imperfection can be clearly separated in the final verification. In some very simple cases, where the position of the critical cross-section $m$ could be directly determined, the effect of the $2^{\text {nd }}$ order analysis on the imperfect structure could be calculated by just using the simplified formula (9). As there are no experimentally established amplitudes for the EUGLI imperfections, the proposed procedure for determining the $e_{0, d, m}$, which is based on the substitute member method, has to be used. This step might be confusing for EC users, who may be unfamiliar with the background of the EUGLI method.
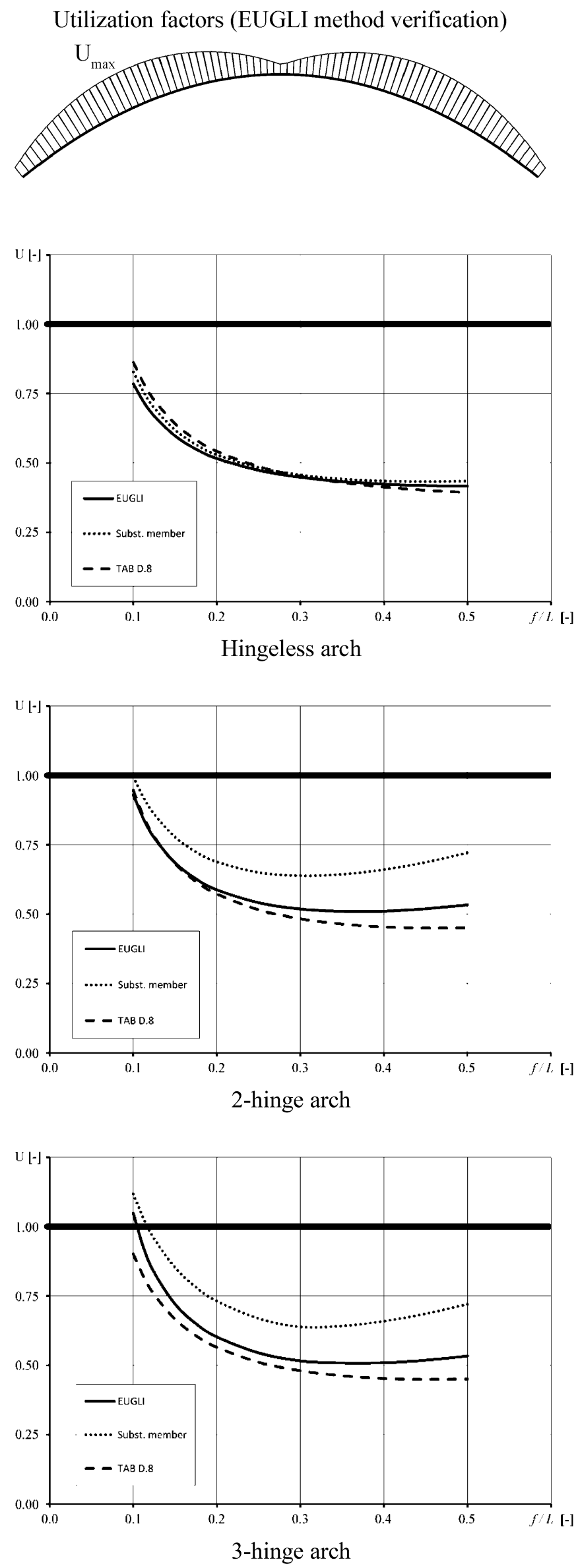

Fig. 5-7 Results of the parametric study. 


\section{REFERENCES}

STN EN 1993-1-1 (2006) Eurocode 3 Design of steel structures. Part 1-1: General rules and rules for buildings, clause 5.3.2 (11) Imperfections for global analysis of frames,SÚTN, pp. 33-36.

STN EN 1993-2 (2003) Eurocode 3 Design of steel structures. Part 2: Steel bridges. Annex D: Buckling lengths of members in bridges and assumptions for geometrical imperfections, D.3: Arched bridges. SÚTN, pp. 96-100.

Chladný E. - Štujberová M. (2013) The equivalent unique global and local imperfection in the shape of the elastic critical buckling mode for the verification of the buckling resistance of compressed members and frame structures using second order analysis, A two-part article. Stahlbau (in print), Oct.-Nov.

Kováč M. (2012) Buckling resistance of metal members and frames. Applications of new methods from the Eurocodes (in Slovak), Slovak University of Technology Publishing. Bratislava, pp. 75-92.

Baláž I. (2008) Determination of the flexural buckling resistance of frames with members with non-uniform cross-section and non-uniform axial compression forces, Proceedings from $34^{\text {th }}$ meeting of the steelwork section workers with foreign attendance 'Theoretical and constructional problems of the steel and timber constructions and bridges', 16-17 Oct. 2008, Pezinok, pp. 17-22.
Baláž I. (2009) Resistance of metal frames with UGLI imperfections, XII. International scientific conference, $110^{\text {th }}$ anniversary of FAST VUT establishment in Brno, Brno, 20-22 April 2009, Section: Engineering constructions, pp. 11-14.

Baláž I.- Koleková Y. (2010) Metal frames with non-uniform members and/or non-uniform normal forces with imperfections in the form of elastic buckling mode, Engineering Research. Anniversary volume honoring Amália and Miklós Iványi, Pollack Mihaly Faculty of Engineering, University of Pécs, 25-26 Oct. 2010, pp. B:3-B:15.

Baláž I. - Ároch R. - Chladný E. - Kmet' S. - Vičan J. (2010) Design of steel structures according to Eurocodes, STN EN 19931-1:2006 and STN EN 1993-1-8:2007, Slovak Chamber of Civil Engineers (SKSI) Bratislava, $1^{\text {st }}$ ed. May 2007, $2^{\text {nd }}$ ed. Jan. 2010 (in Slovak).

Rubin H. - Aminbaghai M. - Weier H.(2004) Structural analysis software IQ 100, (in German) TU Vienna. 